\title{
Characterization of Polymerization Reagents Incorporated into Poly(methyl methacrylate) Chains by Pyrolysis-Gas Chromatography
}

\author{
Hajime Ohtani, Sohji Ishiguro, Minoru TANAKA, \\ and Shin TSUGE* \\ Department of Chemistry, Faculty of Engineering, \\ Nagoya University, Nagoya 464, Japan
}

(Received August 5, 1988)

\begin{abstract}
High-resolution pyrolysis-gas chromatography was successfully applied to characterize the end groups of poly(methyl methacrylate) (PMMA) prepared by radical polymerization. Although PMMA mainly depolymerized to the monomer at elevated temperatures, some of the minor peaks on the pyrograms could be assigned to the products associated with the end groups derived from both the initiation reactions caused by benzoyl peroxide (BPO) and the chain transfer reactions to dodecanethiol and solvent. Two kinds of BPO-initiated chain ends were clearly distinguished. It was confirmed that phenyl-initiated polymer chains rather than benzoyloxyinitiated ones were preferentially formed in solution polymerization, whereas the latter predominated in bulk polymerization. Moreover, the characteristic peak intensities could be correlated to the amounts of polymerization reagents in the feed. In addition, dodecanethiol formed from thiol-incorporating chain ends was selectively detected without interference from any other pyrolysis products by using a sulfur-specific flame photometric detector.
\end{abstract}

KEY WORDS Pyrolysis-Gas Chromatography / Poly(methyl methacrylate)

/ End Group / Polymerization Reagent / Initiation Reaction / Chain

Transfer Reaction /

The radical polymerization of vinyl monomers is generally induced by the dissociation or decomposition of appropriate initiators. The fragments of the initiators responsible for the polymerization reactions are usually incorporated into the resulting polymer chains as end groups. Moreover, the fragments of solvent and chain transfer reagents added can be also incorporated through chain transfer reactions. The end groups thus formed sometimes have significant effects on the properties of the resulting polymers such as thermal stability. Furthermore, excellent information for understanding the mechanisms of the associated polymerization is implicit in the structures and relative proportions of these end groups. Therefore, the characterization of the polymerization reagents incorporated into the polymer chain ends has been one of the most important subjects in polymer characterization. However, the identification and determination of polymer chain ends are usually not easy tasks mainly because of thier low concentrations in polymer chains.

In addition to the radioactive isotopelabeling method, notably by ${ }^{14} \mathrm{C}$-labeled reagents, NMR has been extensively utilized in the fields of the chain end characterization. ${ }^{1-4}$ In some cases, NMR has been successfully applied to characterize the chain ends of relatively low molecular weight oligomers ${ }^{1}$ and the polymers prepared with isotope-labelled reagents such as ${ }^{13} \mathrm{C}$ enriched initiators ${ }^{1,2}$ and perdeuterated monomers. ${ }^{3}$ The NMR methods often provide significant information not only on the number of initiator fragments 
but also how the fragment is incorporated into the polymer chain. ${ }^{3,4}$

On the other hand, pyrolysis-gas chromatography (PyGC) is a simple but extremely sensitive technique which often provides unique structural supplemental information to that obtained by conventional spectroscopic methods. ${ }^{5.6}$ Particularly, the recent advent of extremely efficient fused-silica capillary columns has greatly improved the resolution and extended the applicability of PyGC to characterizing the microstructures of polymers such as branching in polyethylenes, ${ }^{7}$ disordered configuration in highly alternating copolymers, ${ }^{8}$ junctions of different monomer units in tapered copolymers, ${ }^{9}$ and networks in vulcanized rubbers. ${ }^{10}$

In this work, a new method to characterize the polymerization reagents incorporated into polymer chain is proposed by using highresolution PyGC. By this method, the end groups in poly(methyl methacrylate) (PMMA) radically polymerized with benzoyl peroxide (BPO) as an initiator and dodecanethiol as a chain transfer reagent are investigated. Characteristic peaks on the resulting pyrograms are successfully interpreted in terms of the structures and the amounts of the polymer chain ends. On the basis of the obtained results, the polymerization mechanisms of the PMMA samples are discussed. Furthermore, it is shown that the use of a sulfur-selective flame photometric detector (FPD) is effective for investigating the end groups from polymerization reagents containing sulfur.

\section{EXPERIMENTAL}

\section{Materials}

Methyl methacrylate monomer was obtained commercially and purified by a standard procedure. The monomer and solvent (toluene) were distilled under nitrogen atmosphere with reduced pressure. Polymerization was carried out in a $200 \mathrm{ml}$ three neck flask fitted with a nitrogen inlet on a water bath, the temperature of which was regulated at $100^{\circ} \mathrm{C}$. Five $\mathrm{ml}$ of the monomer, $50 \mathrm{ml}$ of solvent, $0.015-0.075 \mathrm{mg}(0.3-1.5 \mathrm{w} / \mathrm{v} \%$ to monomer $)$ of the initiator (BPO) and $0-0.075 \mathrm{mg}(0-1.5$ $\mathrm{w} / \mathrm{v} \%$ to monomer) of the chain transfer reagent (dodecanethiol) were introduced into the flask and subjected to the polymerization under the nitrogen atmosphere for an hour with stirring by a magnetic stirrer. The reaction mixture was poured into $500 \mathrm{ml}$ of methanol to precipitate the polymer. The polymer thus obtained was reprecipitated from the benzene solution into methanol. The precipitate was collected by filtration, washed several times with methanol, and dried in vacuo at $100^{\circ} \mathrm{C}$ for 24 hours. The yields of the polymer samples were between $5-40 \%$. In addition, another PMMA sample polymerized in bulk with both $0.015 \mathrm{mg}$ of BPO and dodecanethiol and that polymerized thermally without any polymerization reagent were also used. Ten kinds of PMMA samples thus obtained are listed in Table I along with feeds of the polymerization reagents and the number average molecular weight $\left(\bar{M}_{n}\right)$ estimated using gel permiation chromatography (GPC) with polystyrene standards.

Table I. PMMA samples

\begin{tabular}{|c|c|c|c|}
\hline \multirow{2}{*}{ Sample } & $\mathrm{BPO} / \mathrm{MMA}$ & $\mathrm{C}_{12} \mathrm{H}_{25} \mathrm{SH} / \mathrm{MMA}$ & $\bar{M}_{n}^{\mathrm{a}}$ \\
\hline & $w / v \%$ & $\mathrm{w} / \mathrm{v} \%$ & $\times 10^{4}$ \\
\hline M-1 & 0.3 & 0 & 3.5 \\
\hline M-2 & 0.5 & 0 & 3.0 \\
\hline M-3 & 1.0 & 0 & 2.5 \\
\hline M-4 & 1.5 & 0 & 2.3 \\
\hline M-5 & 0.3 & 0.3 & 3.2 \\
\hline M-6 & 0.3 & 0.6 & 3.0 \\
\hline M-7 & 0.3 & 1.0 & 2.3 \\
\hline M-8 & 0.3 & 1.5 & 2.5 \\
\hline$M-9^{b}$ & 0.3 & 0.3 & 13.3 \\
\hline$M-10^{b}$ & 0 & 0 & 43.4 \\
\hline
\end{tabular}

\footnotetext{
a Determined by GPC.

b Bulk polymerization.
} 
Conditions for PyGC

A vertical microfurnace-type pyrolyzer (Yanagimoto GP-1018) was directly attached to a gas chromatograph (Shimadzu GC-7A) with a flame ionization detector (FID) and a flame photometric detector (FPD). ${ }^{10}$ About $0.5 \mathrm{mg}$ of the polymer sample was pyrolyzed under the flow of nitrogen carrier gas. The pyrolysis temperature was set empirically at $460^{\circ} \mathrm{C}$ because lower temperatures caused significant peak broadening and poorer reproducibility of the resulting pyrograms whereas the characteristic fragments reflecting the end groups were missing at higher temperatures. A fused silica capillary column $(50 \mathrm{~m} \times 0.2 \mathrm{~mm}$ i.d.) coated with polydimethylsiloxane $(0.33$ $\mu \mathrm{m}$ thick) immobilized through chemical cross-linking was used. The $50 \mathrm{ml} \mathrm{min}^{-1}$ carrier gas flow-rate at the pyrolyzer was reduced to $0.7 \mathrm{ml} \mathrm{min} \mathrm{m}^{-1}$ at the capillary column by a splitter. The column temperature was initially set at $0^{\circ} \mathrm{C}$ by using a $\mathrm{CO}_{2}$ cooling unit, then programmed to $250^{\circ} \mathrm{C}$ at the rate of $4 \mathrm{C}$ $\mathrm{min}^{-1}$. Identification of the peaks on the pyrograms was carried out using a gas chromatograph-mass spectrometer (Shimadzu QP-1000) with both electron impact (EI) and chemical ionization (CI) sources to which the pyrolyzer was also directly attached.

\section{RESULTS AND DISCUSSION}

\section{Pyrogram of PMMA Polymerized with BPO} and Dodecanethiol in Toluene

Figure 1 shows the pyrogram of the sample M-8 prepared in the presence of BPO and dodecanethiol in toluene along with that of the sample M-10 thermally polymerized without any polymerization reagents. Since PMMA has a tendency to depolymerize mostly into the original monomer at elevated temperatures around $500^{\circ} \mathrm{C}$, the main pyrolysis product on the pyrograms (more than 90\%) was the MMA monomer. In addition, various minor products were observed as well-separated peaks. These are summarized in Table II along with their molecular weights and structures estimated by PyGC-MS measurements. Among these, peaks $\mathrm{A}-\mathrm{I}$ are not observed on the pyrogram of M-10. Therefore, they can be assigned to the fragments of the polymerization reagents incorporated into the polymer chain.

The dissociation of BPO can yield both benzoyloxy and phenyl radicals ${ }^{11}$;

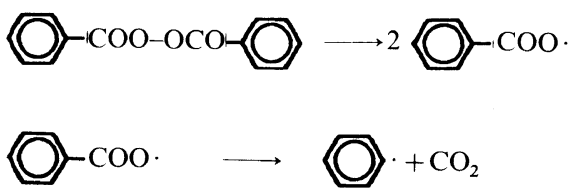

and both radicals cause the initiations reaction as follows:
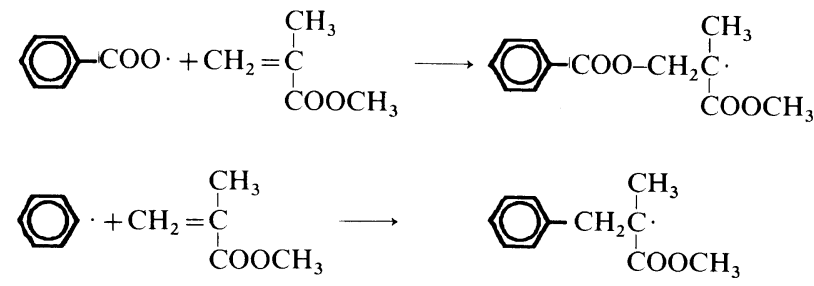

Furthermore, chain transfer from polymeric radicals to solvent (toluene) can also take place:

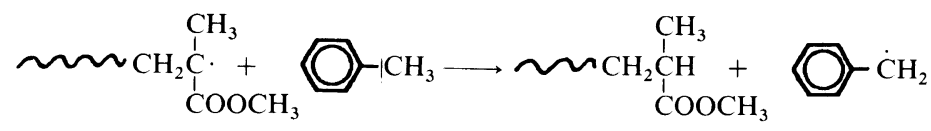




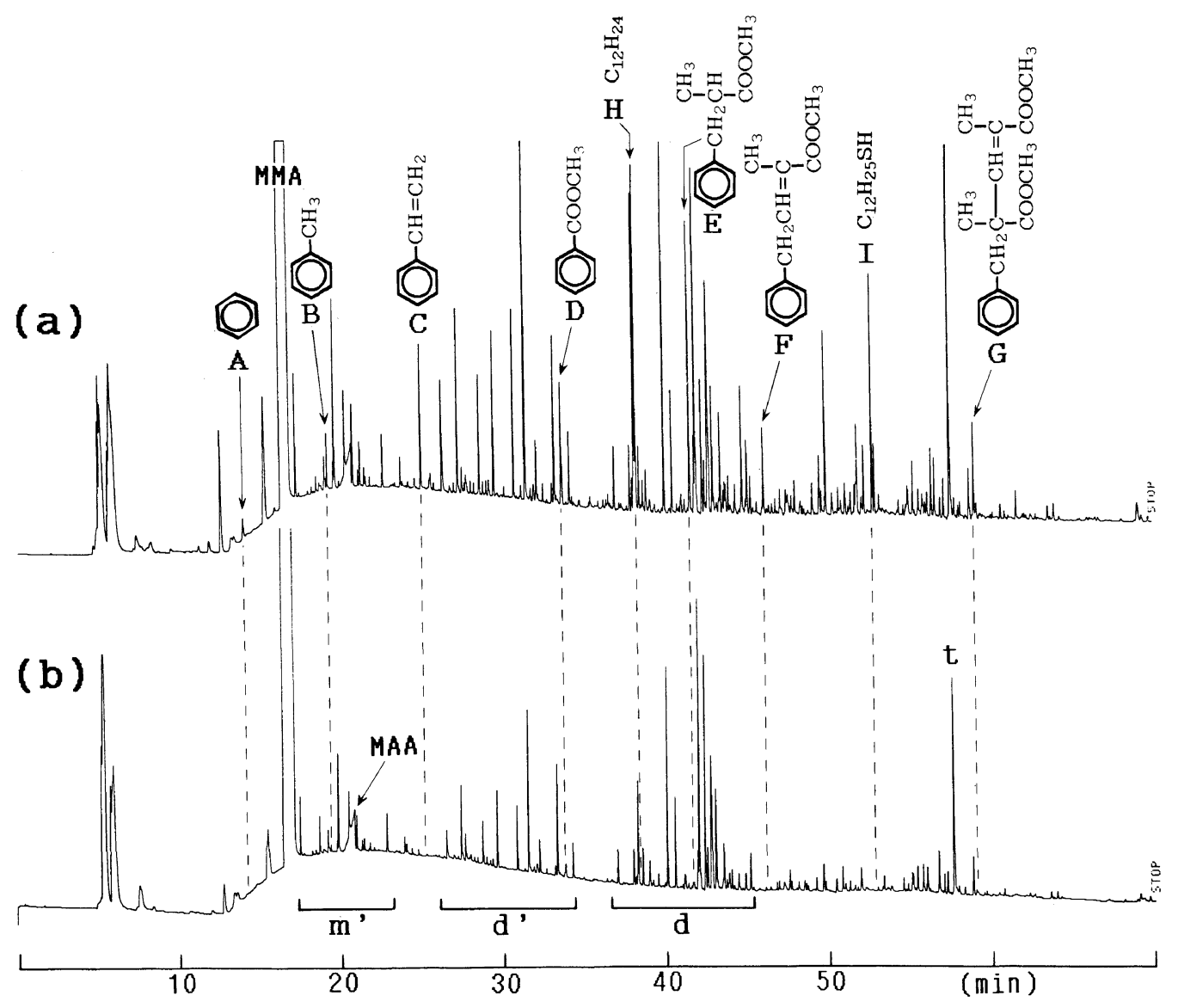

Figure 1. Pyrograms of poly(methyl methacrylate): (a) prepared in toluene with $0.3 \%$ of benzoyl peroxide and $1.5 \%$ of dodecanethiol; (b) thermally polymerized in bulk without any polymerization reagent. Peak assignments are listed in Table II.

The solvent-derived benzyl radical can initiate a further polymerization reaction:

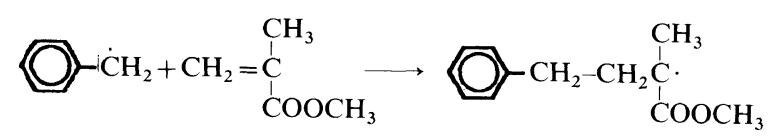

As a result, at least three types of aromatic chain ends should exist in the PMMA samples:

$\mathrm{A}_{1}$<smiles>CCCC(C)(COC(=O)c1ccccc1)C(=O)OC</smiles>
$\mathrm{A}_{2}$<smiles>CCCC(C)(Cc1ccccc1)C(=O)OC</smiles>

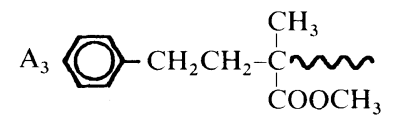

The aromatic products observed on the pyrograms (peaks $A-G$ ) can be attributed to these aromatic chain ends. Methyl benzoate (peak D) should be responsible for the benzoyloxyinitiated chain $\left(A_{1}\right)$ while peaks $E$ and $G$ for the phenyl-initiated chain $\left(\mathrm{A}_{2}\right)$. On the other 
Table II. Peak assignment in the pyrograms of PMMA estimated by PyGC-MS measurements ${ }^{\mathrm{a}}$

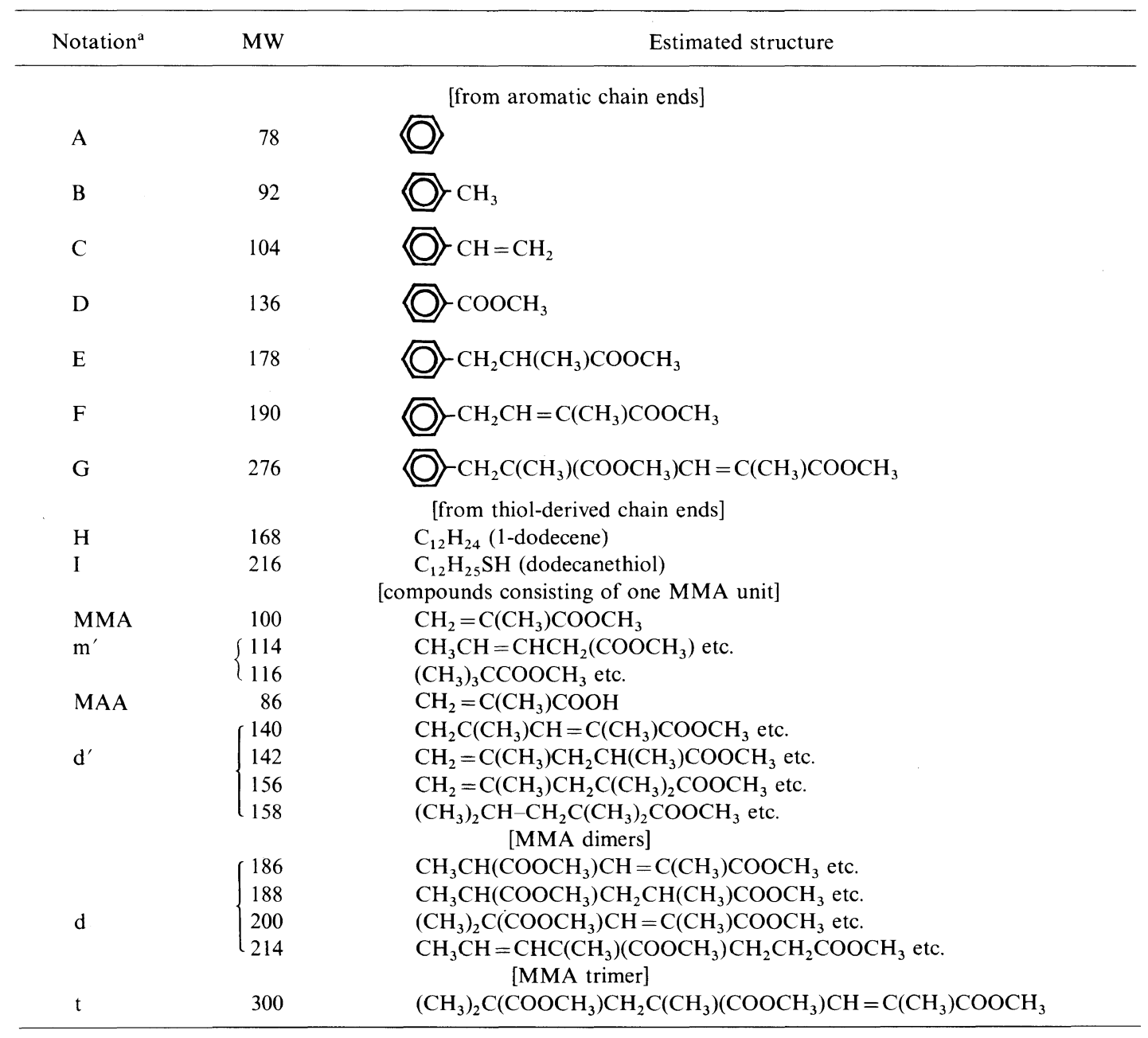

a Peak notations correspond to those in Figure 1.

hand, peaks $\mathrm{C}$ (styrene) and $\mathrm{F}$ can be attributed to the benzyl-initiated chain $\left(\mathrm{A}_{3}\right)$. These products, in fact, are observed exclusively on the pyrograms of the samples prepared in toluene. ${ }^{12}$ Benzene (peak A) might be pro- duced from any types of aromatic chain ends whereas toluene (peak B) can be formed from either $A_{2}$ or $A_{3}$ chain.

Dodecanethiol usually act as a chain transfer reagent as follows:<smiles>CCCCCCCCC(C)C(=O)OC</smiles>

The radical derived from thiol also initiates further polymerization.

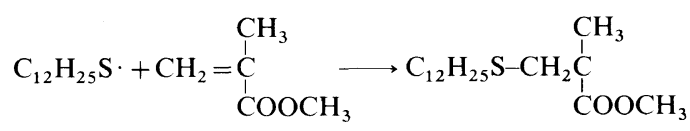


The peaks of 1-dodecene $(\mathrm{H})$ and dodecanethiol (I) on the pyrograms reflect the thiolincorporating chain ends.

The other peaks also observed on the pyrogram of the thermally polymerized PMMA are assigned to the products comprised of only MMA units such as dimers, trimers and their isomers. These products are mainly formed from MMA main chains. Moreover, they might retain various information with respect to the abnormal groups in the polymer chains such as unsaturated terminal groups and head-to-head linkages.

Comparison of Samples Polymerized by Different Methods

Figure 2 shows the pyrograms of PMMA polymerized with $0.3 \%$ of BPO (a) in toluene and (b) in bulk. The products arising from solvent-derived chain end (peak $\mathrm{C}$ and $\mathrm{F}$ ) are naturally not observed on the pyrogram of the bulk-PMMA. Furthermore, the peaks characteristics of the phenyl-initiated chain ends ( $E$ and $G$ ) are much larger on the pyrogram of the solvent-PMMA than in that of the bulkPMMA although the feeds of the initiator were the same for both polymers. On the other hand, the peak of methyl benzoate (D) reflecting the benzoyloxy-initiated chain ends are observed in both pyrograms with similar intensity.

This fact suggests that the phenyl radicals play an important role in the initiation of solution polymerization whereas bulk polym-

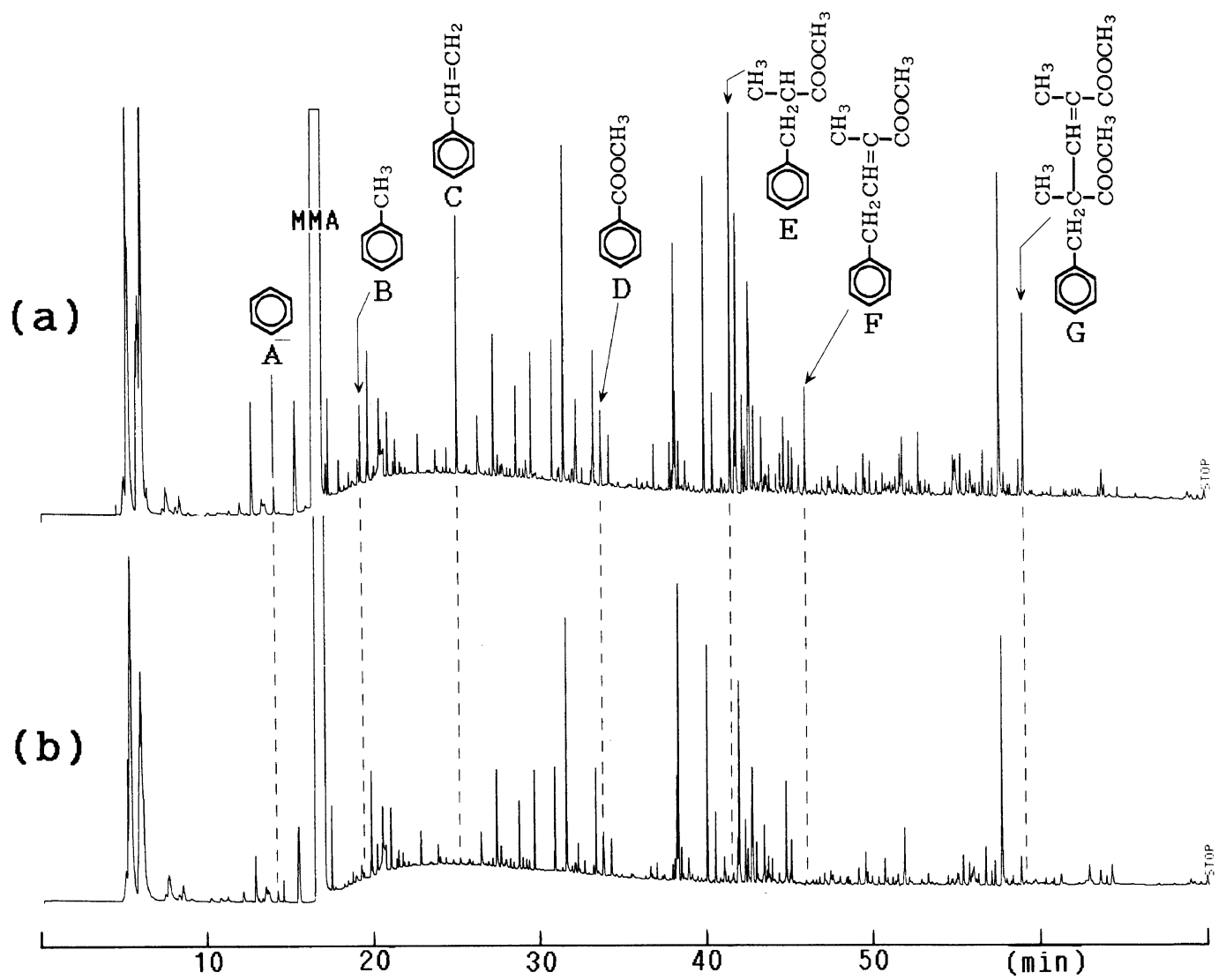

Figure 2. Pyrograms of PMMA prepared with $0.3 \%$ of BPO: (a) by solution polymerization in toluene; (b) bulk polymerization. A-I correspond to those in Figure 1 and Table II. 
erization is predominantly initiated by the benzoyloxy radicals. These results are consistent with those obtained from NMR measurements by Hatada et al. . $^{3,4}$

\section{Relationships between Characteristic Peak}

Intensities and Amounts of Added Polymerization Reagents

In previous sections, it has been demonstrated that the structures of various chain ends derived from polymerization reagents can be identified from characteristic peaks on the resulting pyrograms of PMMA. Furthermore, the relative amount of each end group should be reflected in the intensities of the corresponding peaks. Figure 3 shows the relationships between the amounts of BPO in feed and the relative peak intensities of the characteristic aromatic products for samples $\mathrm{M}-1-\mathrm{M}-4$. The intensities of peaks $E$ and $G$, which are characteristic of phenyl-initiated chain, clearly increase with increase in the amounts of BPO. However, the intensity of methyl benzoate (peak D) are not appreciably dependent on the BPO amount. These phenomena suggest that the ratio of phenyl-initiated chain ends to benzoyloxy-initiated ones increases with increase in the BPO amount. On the other hand, changes in the intensities of peak $C$, characteristic of solvent-derived chain ends, are small because the relative concentration of solvent is almost constant under the the given polymerization conditions.

Figure 4 shows the relationships between the amounts of dodecanethiol in the feed and relative intensities of peaks $\mathrm{H}$ and I characteristic of the thiol-incorporating chain ends for the samples $\mathrm{M}-5-\mathbf{M}-8$. The fact that approximately linear relationships exist between the characteristic peak intensities and the thiol amount suggests that the degree of chain transfer to thiol is nearly proportional to the thiol concentration in the given polymerization reactions.

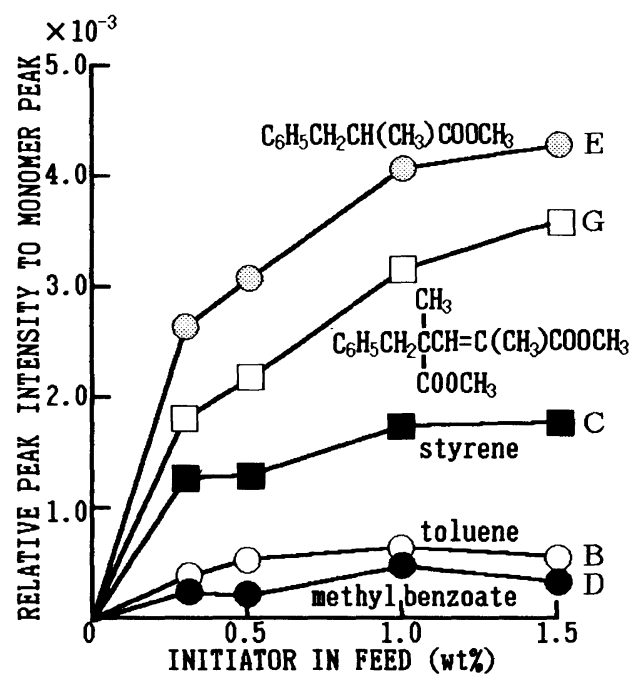

Figure 3. Relationships between relative peak intensities of characteristic aromatic products and the feed $(\%)$ of BPO. B-E and G correspond to those in Figure 1 and Table II.

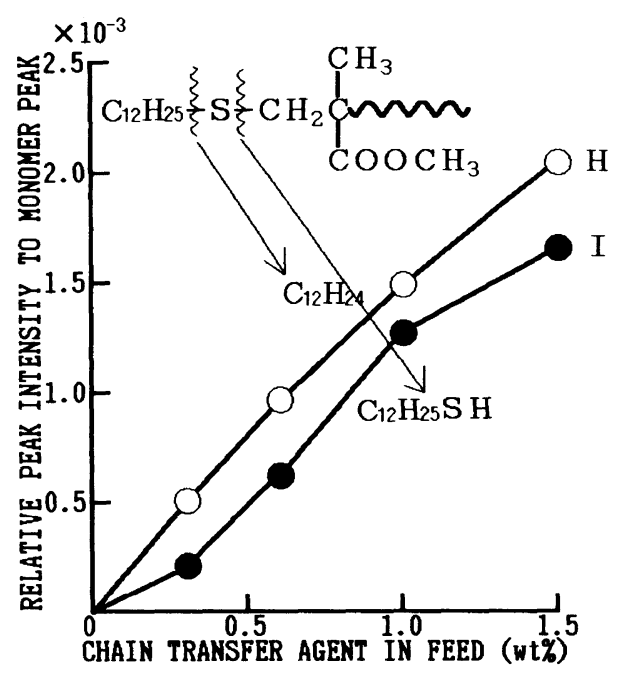

Figure 4. Relationships between relative peak intensities of characteristic $\mathrm{C}_{12}$-products and the feed $(\%)$ of dodecanethiol. $\mathrm{H}$ and $\mathrm{I}$ correspond to those in Figure 1 and Table II.

Selective Detection of Sulfur Compounds from Thiol-Incorporating Chain Ends with FPD

In general, the products characteristic of chain ends are observed as minor peaks on the pyrograms because of the low concentrations 


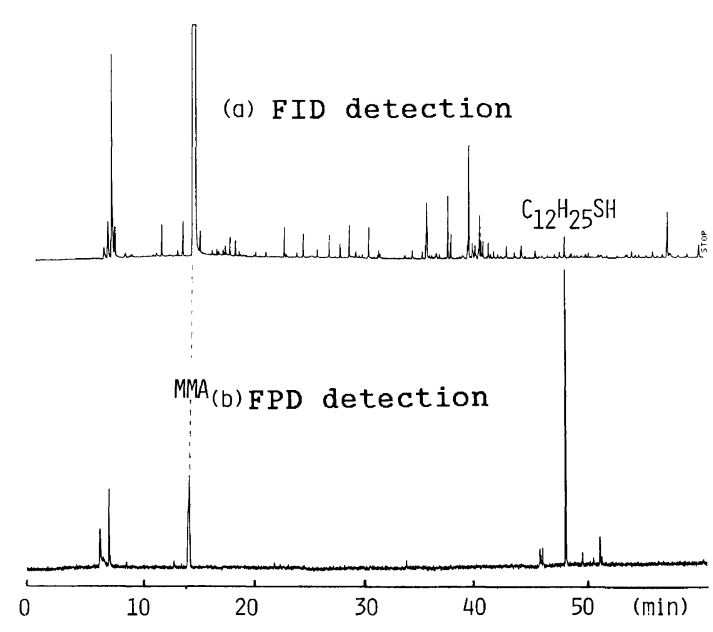

Figure 5. Pyrograms of PMMA prepared with $0.6 \%$ of dodecanethiol: (a) detected by FID; (b) FPD.

of the end groups in high-polymers. The peaks characteristic of chain ends, therefore, are liable to interference of various products formed from polymer main chains. Even in such a case, the characteristic products might be sensitively observed without interference from any other products by using a selective detector for some specific compounds. For example, figure 5 shows the pyrograms of the PMMA prepared in the presence of $0.6 \%$ of dodecanethiol simultaneously detected by an ordinary flame ionization detector (FID) and a sulfur-specific flame photometric detector (FPD). Dodecanethiol formed from thiolincorporating chain ends is selectively detected by FPD while it is observed as one of the minor peaks in the pyrogram detected by FID. Therefore, much smaller amounts of dodecanethiol can be detected by FPD without interference from any other pyrolyzates. The selective detection of specific compounds would be also useful for investigations of nitrogen-, phosphorous- or halogen-containing chain ends by using other selective detectors such as flame thermoionic and electron capture detectors.

Acknowledgement. The authors thank Sumitomo Chemical Industry Co., Ltd. for supplying some PMMA samples. This research was financially supported by a Grant-in-Aid for Scientific Research from the Ministry of Education, Science, and Culture of Japan.

\section{REFERENCES}

1. J. C. Bevington, J. R. Ebdon, and T. N. Huckerby, Eur. Polym. J., 21, 685 (1985).

2. D. E. Axelson and K. E. Russell, Prog. Polym. Sci., 11, 221 (1985).

3. K. Hatada, T. Kitayama, and E. Masuda, Polym. J., 18, 395 (1986).

4. T. Kashiwagi, A. Inaba, J. E. Brown, K. Hatada, T. Kitayama, and E. Masuda, Macromolecules, 19, 2160 (1986).

5. H. Ohtani and S. Tsuge, "Applied Polymer Analysis and Characterization," J. Mitchell, Jr., Ed., Hanser Publishers, Munich, 1987, p 217.

6. S. Tsuge, Chromatogr. Forum, 1, 44 (1986).

7. H. Ohtani, S. Tsuge, and T. Usami, Macromolecules, 17, 2557 (1984).

8. S. Tsuge, T. Kobayashi, Y. Sugimura, T. Nagaya, and T. Takeuchi, Macromolecules, 12, 988 (1979).

9. S. Tsuge, Y. Sugimura, T. Kobayashi, T. Nagaya, and H. Ohtani, "Polymer Characterization," Adv. Chem. Ser. 203, Am. Chem. Soc., Washington, D.C., 1983, p 625 .

10. H. Nakagawa, S. Tsuge, and K. Murakami, J. Anal. Appl. Pyrolysis, 10, 31 (1986).

11. D. H. Solomon, J. Macromol. Sci.-Chem., A17, 337 (1982).

12. H. Ohtani, M. Tanaka, and S. Tsuge, J. Anal. Appl. Pyrolysis, in press. 